\title{
A combined zone-LP and simulated annealing algorithm for unequal-area facility layout problem
}

\author{
Xiao, Y.J. ${ }^{a, b}$, Zheng, Y. ${ }^{c}$, Zhang, L.M. ${ }^{d,}{ }^{*}$, Kuo, Y.H. ${ }^{\mathrm{e}}$ \\ a Jiangsu Key Laboratory of Modern Logistics, School of Marketing and Logistic Management, Nanjing University of Finance \\ and Economics, Nanjing , China \\ ${ }^{\mathrm{b}}$ Business school, Nanjing University, Nanjing, China \\ 'College of Automobile and Traffic Engineering, Nanjing Forestry University, Nanjing, China \\ ${ }^{\mathrm{d} S c h o o l}$ of Management and Engineering, Nanjing University, Nanjing, China \\ e Stanley Ho Big Data Decision Analytics Research Centre, The Chinese University of Hong Kong, Shatin, New Territories, \\ Hong Kong
}

\section{A B S T R A C T}

Facility layout problem (FLP) is one of well-known NP-hard problems and has been demonstrated to be useful in enhancing the productivity of manufacturing systems in practice. This paper focuses on the unequal-area FLP (UA-FLP) whose goal is to locate departments with different areas within a given facility so as to minimize the total material handling cost. A novel approach, which we call a combined zone-linear programming (zone-LP) and simulated annealing algorithm, is developed for solving the UA-FLP. The zone-LP approach is a layout construction technique for the unequal-area departments and consists of two phases. In the first phase, a zoning algorithm is implemented to determine the relative positions between the departments. In this algorithm, for the sake of problem simplification and computational efficiency, each department is treated as a rectangle with an allowable aspect ratio and the area of the facility is assumed to be unbounded. In the second phase, by using the relative positions obtained in the first phase as input, a linear programming (LP) model is developed to identify the exact locations and dimensions of departments within the facility with specified sizes while satisfying their maximum aspect ratio requirement and the shape constraints. We also design a simulated annealing algorithm to improve the placing sequence. Finally, our computational results suggest that our proposed algorithm is efficient compared with the best existing approach in the literature.
\end{abstract}

\section{ARTICLE INFO}

Keywords:

Facility layout problem

Unequal area

Zone-LP approach

Simulated annealing

*Corresponding author:

zhanglm@nju.edu.cn

(Zhang, L.M.)

Article history:

Received 10 August 2016

Revised 9 October 2016

Accepted 15 October 2016

\section{References}

[1] Tompkins, J.A., White, J.A., Bozer, Y.A., Tanchoco, J.M.A. (2010). Facilities Planning, $4^{\text {th }}$ edition, John Wiley \& Sons, New York, USA.

[2] Kusiak, A., Heragu, S.S. (1987). The facility layout problem, European Journal of Operational Research, Vol. 29, No. 3, 229-251, doi: 10.1016/0377-2217(87)90238-4.

[3] Meller, R.D., Gau, K.Y. (1996). The facility layout problem: Recent and emerging trends and perspectives, Journal of Manufacturing Systems, Vol. 15, No. 5, 351-366, doi: 10.1016/0278-6125(96)84198-7.

[4] Singh, S.P., Sharma, R.R.K. (2006). A review of different approaches to the facility layout problems, The International Journal of Advanced Manufacturing Technology, Vol. 30, No.5-6, 425-433, doi:10.1007/s00170-005-0087-9.

[5] Drira, A., Pierreval, H., Hajri-Gabouj, S. (2007). Facility layout problems: A survey, Annual Review in Control, Vol. 31, No. 2, 255-267, doi: 10.1016/j.arcontrol.2007.04.001.

[6] Kulturel-Konak, S., Konak, A. (2011). Unequal area flexible bay facility layout using ant colony optimisation, International Journal of Production Research, Vol. 49, No.7, 1877-1902, doi: 10.1080/00207541003614371. 
[7] Jolai, F., Tavakkoli-Moghaddam, R., Taghipour, M. (2012). A multi-objective particle swarm optimisation algorithm for unequal sized dynamic facility layout problem with pickup/drop-off locations, International Journal of Production Research, Vol. 50, No. 15, 4279-4293, doi: 10.1080/00207543.2011.613863.

[8] Navidi, H., Bashiri, M., Bidgoli, M.M. (2012). A heuristic approach on the facility layout problem based on game theory, International Journal of Production Research, Vol. 50, No. 6, 1512-1527, doi: 10.1080/00207543.2010. $\underline{550638}$.

[9] Ficko, M., Palcic, I. (2013). Designing a layout using the modified triangle method, and genetic algorithms, International Journal of Simulation Modelling, Vol. 12, No. 4, 237-251, doi: 10.2507/IJSIMM12(4)3.244.

[10] Ficko, M., Brezovnik, S., Klancnik, S., Balic, J., Brezocnik, M., Pahole, I. (2010). Intelligent design of an unconstrained layout for a flexible manufacturing system, Neurocomputing, Vol. 73, No. 4-6, 639-647, doi: 10.1016/ j.neucom.2009.06.019.

[11] Montreuil, B., 1991. A modelling framework for integrating layout design and flow network design, Progress in Materials Handling Research, Vol. 2, 95-115, doi: 10.1007/978-3-642-84356-3 8.

[12] Meller, R.D., Narayanan, V., Vance, P.H. (1998). Optimal facility layout design, Operations Research Letters, Vol. 23, No. 3-5, 117-127, doi: 10.1016/S0167-6377(98)00024-8.

[13] Sherail, H.D., Fraticelli, B.M.P., Meller, R.D. (2003). Enhanced model formulations for optimal facility layout, $O p$ erations Research, Vol. 51, No. 4, 629-644, doi: 10.1287/opre.51.4.629.16096.

[14] Meller, R.D., Chen, W., Sherali, H.D. (2007). Applying the sequence-pair representation to optimal facility layout design, Operations Research Letters, Vol. 35, No. 5, 651-659, doi: 10.1016/i.orl.2006.10.007.

[15] Kim, J.G., Kim, Y.D. (2000). Layout planning for facilities with fixed shapes and input and output points, International Journal of Production Research, Vol. 38, No. 18, 4635-4653, doi: 10.1080/00207540050205550.

[16] Das, S.K. (1993). A facility layout method for flexible manufacturing systems, International Journal of Production Research, Vol. 31, No. 2, 279-297, doi: 10.1080/00207549308956725.

[17] Rajasekharan, M., Peters, B.A., Yang, T. (1998). A genetic algorithm for facility layout design in flexible manufacturing systems, International Journal of Production Research, Vol. 36, No. 1, 95-110, doi: 10.1080/00207549 8193958.

[18] Scholz, D., Petrick, A., Domschke, W. (2009). STaTS: A Slicing Tree and Tabu Search based heuristic for unequal area faciltiy layout problem, European Journal of Operational Research, Vol. 197, No. 1, 166-178, doi: 10.1016/ j.ejor.2008.06.028.

[19] Komarudin, Wong, K.Y. (2010). Applying ant system for solving unequal area facility layout problems, European Journal of Operational Research, Vol. 202, No. 3, 730-746, doi: 10.1016/i.ejor.2009.06.016.

[20] Liu, Q., Meller, R.D. (2007). A sequence-pair representation and MIP model based heuristic for the facility layout problem with rectangular departments, IIE Transactions, Vol. 39, No. 4, 337-394, doi: 10.1080/07408170600 844108.

[21] Bozer, Y.A., Wang, C.T. (2012). A graph-pair representation and MIP-model-based heuristic for the unequal-area facility layout problem, European Journal of Operational Research, Vol. 218, No. 2, 382-391, doi: 10.1016/ j.ejor.2011.10.052.

[22] Xiao, Y., Seo, Y., Seo, M. (2013). A two-step heuristic algorithm for layout design of unequal-sized facilities with input/output points, International Journal of Production Research, Vol. 51, No. 14, 4200-4222, doi: 10.1080/ $\underline{00207543.2012 .752589 .}$. 


\title{
Kombiniran algoritem c-LP in simuliranega ohlajanja za problem razporeditve prostorov neenakih površin znotraj objekta
}

\author{
${ }^{a}$ Jiangsu Key Laboratory of Modern Logistics, School of Marketing and Logistic Management, Nanjing University of Finance \\ and Economics, Nanjing, China \\ ${ }^{\mathrm{b}}$ Business school, Nanjing University, Nanjing, China \\ ${ }^{c}$ College of Automobile and Traffic Engineering, Nanjing Forestry University, Nanjing, China \\ ${ }^{\mathrm{d} S}$ School of Management and Engineering, Nanjing University, Nanjing, China \\ ${ }^{\text {e}}$ Stanley Ho Big Data Decision Analytics Research Centre, The Chinese University of Hong Kong, Shatin, New Territories, \\ Hong Kong
}

\section{POVZETEK}

Problem razporeditve prostorov je znan računsko zahteven problem in se s pridom uporablja za povečanje produktivnosti proizvodnih sistemov. Ta članek se ukvarja z znižanjem stroškov pretoka materiala, z razporeditvijo prostorov $\mathrm{v}$ proizvodnih objektih, $\mathrm{v}$ katerih oddelki nimajo enakih površin. Za rešitev problema je vpeljan nov algoritem, ki je kombinacija conskega linearnega programiranja (c-LP) in simuliranega ohlajanja. Algoritem c-LP je primeren za razporeditev prostora za oddelke neenakih površin in je dvofazen. $V$ prvi fazi je uporabljen conski algoritem s katerim so pridobljene relativne razdalje med oddelki. Oddelki so poenostavljeno obravnavani kot pravokotniki z nastavljivim razmerjem stranic, velikost celotnega objekta pa ni omejena. V drugi fazi inovativni model linearnega programiranja iz pridobljenih razdalj med oddelki in omejitev oblik oddelkov pridobi natančne pozicije in dimenzije oddelkov znotraj objekta. Za izboljšanje zaporedja postavitve oddelkov je uporabljen algoritem simuliranega ohlajanja. Računsko pridobljeni rezultati potrjujejo učinkovitost predlaganega pristopa $\mathrm{v}$ primerjavi $\mathrm{z}$ najboljšimi obstoječimi pristopi.
\end{abstract}

(C) 2016 PEI, University of Maribor. All rights reserved.

\section{PODATKI O ČLANKU}

Ključne besede:

Problem razporeditve prostorov

Neenake površine

c-LP pristop

Simulirano ohlajanje

*Kontaktna oseba: srimbo@mek.dtu.dk (Boorla, S.M.)

Zgodovina članka:

Prejet 10. avgusta 2016

Popravljen 9. oktobra 2016

Sprejet 15. oktobra 2016 